CLINICAL REPORT

T.J. Tan

T.Y. Tan

\section{CT Features of Parotid Gland Oncocytomas: A Study of 10 Cases and Literature Review}

SUMMARY: Oncocytomas of the salivary glands are rare benign epithelial tumors which occur most commonly in the parotid gland. The aim of our study was to characterize the clinical-radiologicpathologic spectrum of parotid oncocytomas in a series of 10 cases seen in our institution between January 2003 and November 2008. The CT features of parotid oncocytomas in the largest imaging series of this rare but important benign lesion include a well-defined enhancing tumor with a "deformable" appearance when large, and a non-enhancing curvilinear cleft or cystic component. These CT findings are potentially helpful in distinguishing these benign lesions from other parotid tumors in clinical scenarios that preclude surgical resection or when biopsy results are non-diagnostic. Further studies are advocated to validate the specificity and positive predictive value of these imaging features. $\mathbf{0}$ ncocytomas of the salivary gland, which were first described by Jaffé in 1932, are benign epithelial tumors that occur most commonly in adults in their sixth to eighth decades, ${ }^{1-6}$ with no clear sex predilection. ${ }^{3,4,6-9}$ These neoplasms are histologically composed of monotonous sheets of oncocytes, epithelial cells named by Hamperl in 1931, which are characterized by an intensely eosinophilic and granular cytoplasm. These metabolically altered epithelial cells accumulate numerous cytoplasmic mitochondria through the process of oncocytic metaplasia. ${ }^{1,4,5,7,10-12}$ Synonyms for this tumor include oncocytic adenoma, oxyphilic granular-cell adenoma, and oxyphilic adenoma.

Oncocytomas are rare, comprising just $0.5 \%-1.5 \%$ of salivary gland tumors. ${ }^{4,7,9,13-16}$ The parotid gland is the most commonly involved salivary gland, accounting for $78 \%-84 \%$ of salivary gland oncocytomas. ${ }^{2,3}$ Oncocytomas occurring in the submandibular gland and the minor salivary glands have been reported but are relatively uncommon., ${ }^{2,7,9}$

$\mathrm{CT}$ is one of the established first-line imaging modalities used in the assessment of major salivary gland tumors. ${ }^{2,17,18}$ To the authors' knowledge, there is limited description of the CT imaging features of parotid gland oncocytomas in the published literature, apart from a few case reports. ${ }^{15,16,19-21}$

Because the surgical resection of parotid tumors is generally the treatment of choice and allows histopathologic confirmation, the identification of specific CT imaging features suggestive of benign parotid oncocytomas will become helpful in terms of simply managing the patient expectantly when certain clinical scenarios arise-for example, if the patient declines surgical intervention, when patient factors such as age and multiple comorbidities preclude surgical resection, or when biopsy results are not diagnostic. In this study, we describe the CT features of 10 histopathologically proved cases of parotid oncocytomas, the largest imaging series in the published literature, to our knowledge.

\section{Materials and Methods}

A review of parotid tumors that were discussed at combined clinicalradiologic-pathologic sessions in our institution between January

Received December 2, 2009; accepted after revision February 15, 2010.

From the Department of Diagnostic Radiology, Changi General Hospital, Singapore.

Please address correspondence to Tan Tien Jin, MD, Department of Diagnostic Radiology, Changi General Hospital, 2 Simei St 3, Singapore 529889; e-mail: tienjin.tan@mohh.com.sg DOI 10.3174/ajnr.A2090
2003 and November 2008 revealed 10 cases of histopathologically confirmed parotid gland oncocytomas. The sex, age at diagnosis, clinical presentation, type of surgical resection (superficial versus total parotidectomy), and recurrence during follow-up of all 10 patients were recorded retrospectively. Institutional review board approval was obtained before the study.

All 10 cases were imaged by using the Aquilion multisection CT scanner (Toshiba Medical Systems, Tokyo, Japan), with the scanning level extending from the external auditory canal to the hyoid bone and the scan plane being parallel to the hard palate.

Five of the 10 patients were imaged by using the 4-section Toshiba system. Images were acquired at $135 \mathrm{kV}$ and $250 \mathrm{~mA}$, with a pitch factor of 3.5 and a total scanning time of 30 seconds. Axial sections of 3-mm thickness were reconstructed with use of the FC 10 soft-tissue algorithm. A bolus intravenous dose of $70 \mathrm{~mL}$ of nonionic contrast ( $350 \mathrm{mg} \mathrm{I} / \mathrm{mL}$ ) was administered to patients at an injection rate of 1.5 $\mathrm{mL}$ per second. Scanning was initiated 47 seconds after the onset of contrast injection.

The remaining 5 patients were imaged by using the 64 -section Toshiba system. Images were acquired at $120 \mathrm{kV}$ and $200 \mathrm{~mA}$ with a pitch factor of 0.8 and a total scanning time of 7 seconds. Axial sections of 3-mm thickness were reconstructed with the use of the FC 04 soft-tissue algorithm. A bolus intravenous dose of $50 \mathrm{~mL}$ of nonionic contrast $(350 \mathrm{mg} \mathrm{I} / \mathrm{mL})$ was administered to patients at an injection rate of $1.5 \mathrm{~mL}$ per second. Scanning was initiated 50 seconds after the onset of contrast injection.

A senior head and neck radiologist retrospectively reviewed the CT images of the 10 confirmed cases of parotid gland oncocytomas. The tumors were then categorized in terms of location, size, margins, contour, and pattern of enhancement.

Tumor location was defined as involving the superficial, deep, or both the superficial and deep lobes of the parotid gland. The intraparotid segment of the facial nerve creates a surgical plane that divides the gland into superficial and deep lobes but is not routinely identified even with high-resolution imaging. ${ }^{22}$ The course of the intraparotid facial nerve can be inferred by identification of the retromandibular vein within the gland because the nerve lies just lateral to this vessel. ${ }^{17,22}$ The parotid oncocytomas in our series were classified as involving the superficial lobe if the tumor was located lateral to the retromandibular vein and as involving the deep lobe if the tumor was located medial to the retromandibular vein or showed extension medially through the stylomandibular gap. Each patient was also evaluated for synchronous bilateral and multifocal disease. The size of the tumor was expressed in terms of maximal axial dimensions measured to the nearest millimeter. The largest lesion was measured in cases of 


\begin{tabular}{|c|c|c|c|c|c|c|c|c|}
\hline \multicolumn{9}{|c|}{ Age and sex distribution including the radiologic features of the 10 cases of parotid gland oncocytomas } \\
\hline No. & $\begin{array}{c}\text { Sex/Age } \\
(y r)\end{array}$ & Bilaterality/Multifocality & Location & $\begin{array}{l}\text { Size } \\
(\mathrm{mm})\end{array}$ & Margins & Contour & Enhancement & $\begin{array}{c}\text { Presence of Nonenhancing } \\
\text { Curvilinear Cleft/Cystic } \\
\text { Component }\end{array}$ \\
\hline 1 & $\mathrm{~F} / 55$ & Unilateral/Solitary & Superficial lobe & $11 \times 8$ & Sharp & Lobulated & Heterogeneous & Nonenhancing curvilinear cleft \\
\hline 2 & $\mathrm{~F} / 70$ & Bilateral/Multifocal & Superficial and deep lobes & $32 \times 15$ & Sharp & Smooth & Heterogeneous & Nonenhancing curvilinear cleft \\
\hline 3 & $\mathrm{~F} / 74$ & Bilateral/Multifocal & Superficial and deep lobes & $9 \times 6$ & Sharp & Smooth & Homogeneous & \\
\hline 4 & $\mathrm{~F} / 49$ & Unilateral/Solitary & Superficial lobe & $10 \times 8$ & Sharp & Smooth & Homogeneous & \\
\hline 5 & $\mathrm{M} / 49$ & Unilateral/Solitary & Superficial lobe & $13 \times 12$ & Sharp & Lobulated & Heterogeneous & Cystic component \\
\hline 6 & $\mathrm{M} / 60$ & Bilateral/Multifocal & Superficial and deep lobes & $12 \times 10$ & Sharp & Lobulated & Homogeneous & \\
\hline 7 & $F / 49$ & Unilateral/Solitary & Superficial lobe & $6 \times 6$ & Sharp & Lobulated & Heterogeneous & Cystic component \\
\hline 8 & $F / 68$ & Bilateral/Multifocal & Superficial and deep lobes & $12 \times 8$ & Sharp & Smooth & Heterogeneous & Nonenhancing curvilinear cleft \\
\hline 9 & $\mathrm{~F} / 70$ & Bilateral/Multifocal & Superficial and deep lobes & $66 \times 42$ & Sharp & Smooth & Heterogeneous & Nonenhancing curvilinear cleft \\
\hline 10 & $M / 66$ & Unilateral/Multifocal & Superficial and deep lobes & $27 \times 18$ & Sharp & Smooth & Homogeneous & \\
\hline
\end{tabular}

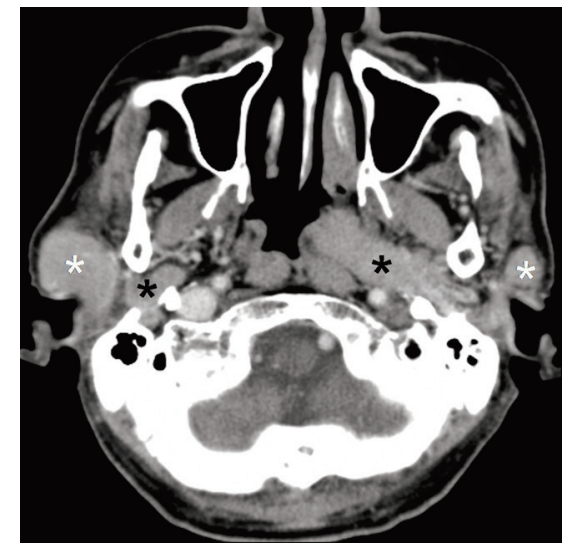

Fig 1. A 60-year-old man with bilateral parotid oncocytomas. CT scan shows bilateral multifocal and homogeneously enhancing parotid tumors with well-defined margins involving both the superficial (white asterisks) and deep (black asterisks) lobes of the parotid gland.

multifocal disease. The tumor was deemed to have sharp margins if it was well-demarcated throughout its circumference and to have indistinct margins otherwise. A tumor was considered to have a lobulated contour if it demonstrated surface undulations or to have a smooth contour if it lacked such undulations.

Because precontrast images were not acquired, a tumor was presumed to be enhancing if it showed a mean attenuation of $>100 \mathrm{HU}$ and appeared significantly more hyperattenuated than the native parotid parenchyma and ipsilateral muscles on visual comparison. The enhancement pattern of the tumors was categorized as either homogeneous or inhomogeneous. Tumors that showed inhomogeneous enhancement were further described as demonstrating either a nonenhancing curvilinear cleft or a cystic component; the latter was defined as having a mean attenuation of $\leq 20 \mathrm{HU}$ and being round or oval in appearance.

\section{Results}

The Table shows the age and sex distribution as well as the radiologic features of the parotid gland oncocytomas. Of the 10 patients, 7 were women and 3 were men between 49 and 74 years of age (mean age, 61 years). All 10 patients initially presented with slowly enlarging painless parotid masses ranging from 6-96 months (mean, 30 months); 2 patients were found clinically to have bilateral parotid masses.

CT revealed a unilateral solitary tumor in 4 patients, unilateral multifocal disease in 1 patient, and synchronous bilateral multifocal tumors (Fig 1) in the remaining 5 patients.

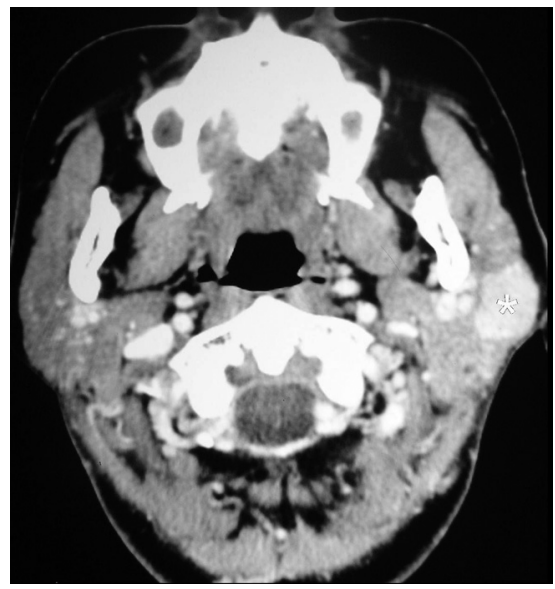

Fig 2. A 49-year-old woman with a unilateral solitary parotid oncocytoma. A well-defined tumor with a lobulated contour and homogeneous enhancement is identified on the CT scan in the superficial lobe of the left parotid gland (white asterisk).

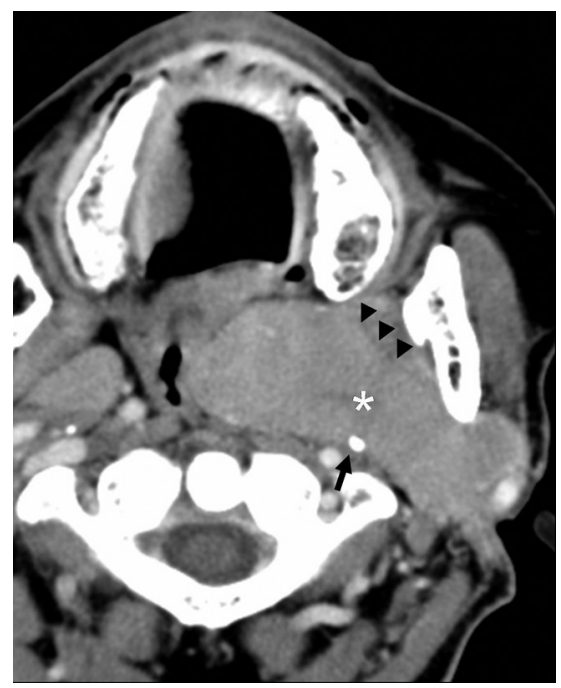

Fig 3. A left parotid oncocytoma in a 68-year-old woman. CT scan reveals a large deformable tumor (white asterisk), which extends medially into the parapharyngeal space through the stylomandibular gap. The contour of the tumor is distorted by the styloid process (black arrow) and the left medial pterygoid muscle (black arrowheads).

Three of the 5 patients found to have bilateral parotid tumors following CT had presented with unilateral parotid masses, and were clinically occult for bilateral disease. The tumor was limited to the superficial lobe of the parotid gland in the 4 patients with unilateral solitary tumors on CT (Fig 2), while 

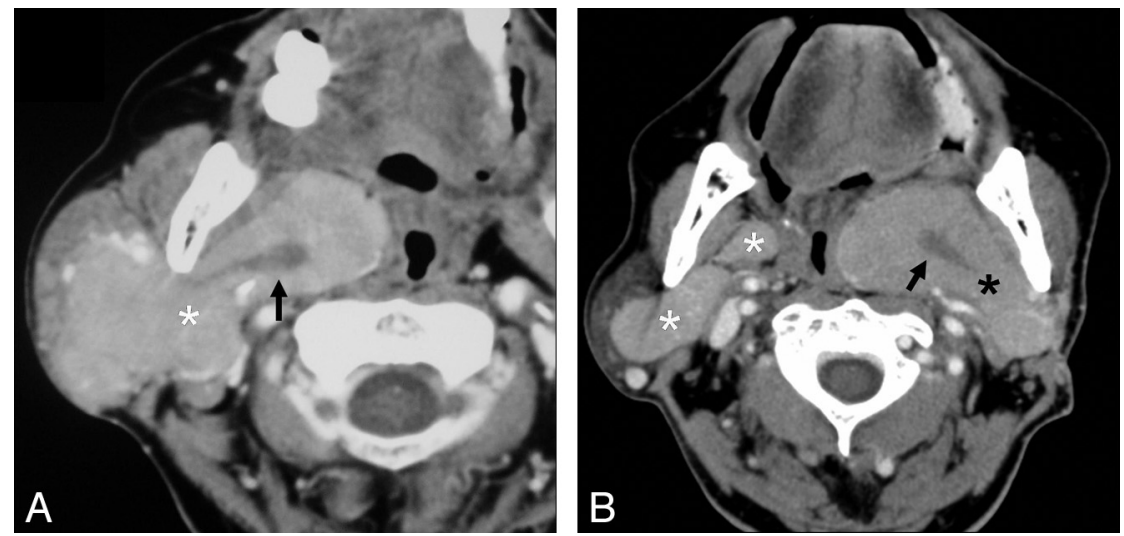

Fig 4. $A, \mathrm{~A}$ 70-year-old woman with a large right parotid oncocytoma. The large tumor (white asterisk) extends medially through the stylomandibular gap into the parapharyngeal space and demonstrates a nonenhancing curvilinear cleft (black arrow). B, Synchronous bilateral parotid oncocytomas in another 70-year-old woman. CT demonstrates tumors in the right parotid gland (white asterisks), which show homogeneous enhancement, and a large left parotid tumor (black asterisk) with a nonenhancing curvilinear cleft (black arrow).

both the superficial and deep lobes were involved in the other 6 patients.

The maximum axial diameter of the tumors ranged from 6 to $66 \mathrm{~mm}$, with a mean of $19.8 \mathrm{~mm}$. All the tumors had sharp margins. The oncocytomas in 4 of the patients demonstrated a lobulated contour, with the tumors in the other 6 patients showing smooth contours. Three patients had large parotid oncocytomas, which involved the deep lobe and showed extension to the parapharyngeal space through the stylomandibular gap. The contours of these large oncocytomas were distorted by surrounding anatomic structures such as the styloid process and surrounding muscles, giving rise to the "deformable" appearance of the tumors (Fig 3).

The tumors in 4 of the patients showed homogeneous enhancement. Heterogeneous enhancement was demonstrated in the remaining 6 patients $(60 \%)$, in whom a central nonenhancing curvilinear cleft was seen in 4 (Fig $4 A,-B$ ) and a cystic component, in the other 2 (Fig 5). None of the tumors showed calcification. Cut sections of the gross specimens of the tumors showing central non-enhancing curvilinear clefts on CT revealed central gray-white scars, which correlated microscopically with hyalinized and fibrous tissue. Cystic degeneration was noted histopathologically with respect to the 2 tumors demonstrated to have nonenhancing cystic components on CT. We concluded that these central scars and areas of cystic degeneration would most likely be responsible for the nonenhancing curvilinear cleft and cystic component detected on CT, respectively.

Seven of the patients in our series underwent either superficial or total parotidectomy, depending on the location of the tumor. Sonography-guided core-needle biopsy was used to obtain the histopathologic diagnosis in the 3 patients who declined surgery.

The follow-up duration of the 7 patients who underwent surgical resection ranged from 12 to 19 months, with a mean of 18 months. At the time of the last follow-up, no clinical evidence of local tumor recurrence was detected in these 7 patients.

\section{Discussion}

The clinical features of oncocytomas resemble those of other benign and low-grade salivary gland tumors making clinical diagnosis challenging. Patients typically present with a solitary slow-growing painless parotid mass, ${ }^{3,4,6,8,9,16,19,20}$ similar to that observed in our series. All 10 patients in our series were

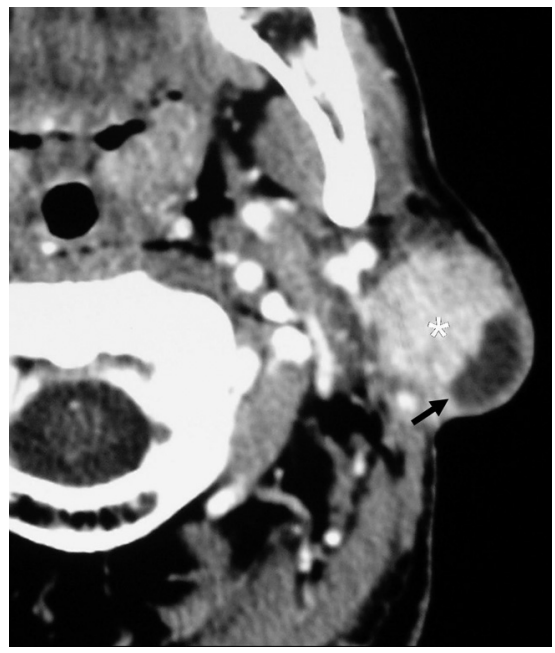

Fig 5. A 49-year-old man with a unilateral solitary parotid oncocytoma. The parotid tumor in the superficial lobe of the left parotid gland (white asterisk) has a lobulated contour and shows a cystic component (black arrow).

adults in their fifth to eighth decades with a female preponderance.

Complete surgical excision of oncocytomas in the form of superficial or total parotidectomy, depending on the location of the tumor, is the treatment of choice, with radiation therapy not indicated because oncocytes are radioresistant. ${ }^{3}$ Local recurrence for oncocytomas following surgery is uncommon, though recurrence rates of $20 \%-22 \%$ have been reported in the literature. ${ }^{4,7-9,23}$ Malignant forms of oncocytomas or oncocytic carcinomas, characterized by cytomorphologically malignant oncocytes or evidence of metastasis, are occasionally reported and account for $<1 \%$ of all salivary gland tumors. ${ }^{1,3,6,14,24,25}$ These are usually associated with a preexisting oncocytoma but may arise de novo. ${ }^{3}$ No histologic features of malignancy were detected in our series, and none of the 7 patients who underwent surgery showed clinical features of disease recurrence following surgery.

Due to their low prevalence, only a few case reports on the CT imaging features of parotid oncocytomas are available in the published literature. ${ }^{15,16,19-21}$ The reports on MR imaging of parotid oncocytomas describe these tumors as demonstrating T1 and T2 hypointensity with homogeneous contrast enhancement. ${ }^{19-21}$ Özcan et al ${ }^{15}$ reported an oncocytoma in the deep lobe of the right parotid gland, which showed T1 hyperintensity, T2 hypointensity, and heterogeneous contrast en- 
hancement. Kasai et $\mathrm{al}^{21}$ described multiple bilateral oncocytomas that were isointense on short $\tau$ inversion recovery imaging and hyperintense on diffusion-weighted imaging, with corresponding low apparent diffusion coefficient values. The tumors also demonstrated early enhancement with early washout on dynamic contrast-enhanced images.

The sonography features of parotid oncocytomas are nonspecific and include a hypoechoic mass with well-defined margins, not unlike other benign parotid tumors such as pleomorphic adenomas. ${ }^{2}$ Parotid oncocytomas have shown focal uptake of technetium Tc99m pertechnetate but not with gallium Ga67m scintigraphy. ${ }^{19-21}$ These tumors have also been reported to demonstrate uptake of fluorodeoxyglucose during positron-emission tomography scanning. ${ }^{26,27}$

The common CT finding of parotid oncocytomas described in the literature is a well-defined parotid mass showing homogeneous enhancement. The important differential diagnoses for well-defined enhancing parotid tumors seen on CT include the Warthin tumor and basal cell adenomas. Warthin tumors show enhancement in the early postcontrast scan phase but decreased enhancement in the delayed phase. ${ }^{28}$ Basal cell adenomas show similar increased enhancement in the early postcontrast scan phase, and gradual washout of contrast in the delayed phase. ${ }^{29}$ Pleomorphic adenomas, the most common parotid tumors, which usually occur in adults older than 40 years of age with a slight female predominance, ${ }^{2,30}$ are considered a less likely differential diagnosis because these demonstrate minimal or no enhancement in the early postcontrast scan phase but progressive enhancement in the delayed phase. ${ }^{28,31}$ A low-grade parotid malignancy is an important differential diagnosis and a major diagnostic pitfall in the imaging assessment of a well-defined enhancing parotid tumor. $2,17,18,28$

In addition, we have reported the features of a nonenhancing curvilinear cleft and a cystic component in the 6 cases of parotid oncocytomas with heterogeneous enhancement in our series. We concluded, by correlating with histology findings, that the nonenhancing curvilinear cleft was most likely secondary to a central scar. The cystic component seen on CT probably corresponded to an area of cystic degeneration, a histologic finding previously associated with oncocytomas. ${ }^{7,9}$ These imaging features have not been previously described in parotid oncocytomas, though Chawla et $\mathrm{al}^{32}$ reported similar linear bands and cystic areas in parotid basal cell adenomas. Shellenberger et $\mathrm{al}^{16}$ described the CT finding of a cystic parotid tail mass, which was confirmed histopathologically as nodular oncocytic hyperplasia, a multifocal process with diffuse oncocytic replacement of the parotid gland that is categorically distinct from an oncocytoma. Cyst formation has also been commonly associated with the Warthin tumors. $^{2,17,28}$

The parotid oncocytomas in all of our patients had sharp margins, which conveyed benignity, in contrast to malignant salivary gland tumors, which usually demonstrate ill-defined margins. ${ }^{2,17}$ The contours of the parotid oncocytomas in 4 of our patients were lobulated, a feature seen mainly with pleomorphic adenomas but also in Warthin tumors and basal cell adenomas. $^{2,17,32,33}$ Three of our patients had large tumors that extended to the parapharyngeal space through the stylomandibular gap. The contours of these tumors were distorted by the surrounding anatomic structures, giving the appearance of deformable tumors, which is similar to the CT findings in the case report of Shellenberger et al. ${ }^{16}$ Rare cases of large parotid deep lobe tumors extending to the parapharyngeal space have been described with Warthin tumor and pleomorphic adenomas. ${ }^{34,35}$

Warthin tumors are usually diagnosed in elderly men, with $10 \%-15 \%$ showing synchronous bilateral disease. ${ }^{17,30,36}$ There is a tendency for oncocytomas to present with synchronous bilateral multifocal disease as evidenced by 5 of 10 patients in our series, with the reported incidence of synchronous bilateral oncocytomas in the reviewed literature ranging from $7 \%$ to $15 \% .^{3,8,23}$

There is, therefore, overlap of radiologic features between oncocytomas and other benign parotid tumors such as Warthin tumors, basal cell adenomas, and, to a lesser degree, pleomorphic adenomas. However, when taken together, the diagnosis of a benign parotid oncocytoma is favored in a middle-aged or elderly woman who presents with CT findings of well-defined, enhancing bilateral, and multifocal parotid tumors that demonstrate a nonenhancing curvilinear cleft. These imaging findings will be atypical for pleomorphic adenomas due to their lack of enhancement in the early postcontrast phase. Bilateral and multifocal parotid tumors on CT also render pleomorphic adenomas and basal cell adenomas less likely differential diagnoses because these tend to present as unilateral solitary tumors.

Although Warthin tumors are commonly associated with cyst formation, they are usually seen in elderly men and have not been reported to demonstrate a nonenhancing curvilinear cleft correlating histologically to a central scar. Large parotid oncocytomas in our series that extended to the parapharyngeal space through the stylomandibular gap exhibited contour distortion by the surrounding anatomic structures. The deformable appearance of these tumors, to our knowledge, has only been rarely reported with other benign parotid tumors in the reviewed literature and may, therefore, be useful in distinguishing parotid oncocytomas on CT.

There are several limitations in our study. The CT findings of the 10 cases of parotid oncocytomas in our study were described by a single unblinded senior head and neck radiologist. The authors acknowledge that a retrospective study in which the CT imaging features of parotid oncocytomas were reported on the basis of consensus by a group of blinded independent observers would have resulted in a reduction in observer bias. Further studies that examine the imaging features of parotid oncocytomas in conjunction with other parotid tumors (both benign and malignant) are necessary to assess the specificity and positive predictive value of the nonenhancing curvilinear cleft and deformable appearance of parotid oncocytomas that were described in our imaging series. A high specificity and positive predictive value of these imaging features for parotid oncocytomas may potentially aid the reporting radiologist in excluding other parotid tumors, particularly malignant neoplasms.

Although there are several reports in the published literature that describe the high sensitivity, specificity, and accuracy of sonography-guided core-needle biopsy in establishing the histopathologic diagnosis for both benign and malignant parotid tumors, ${ }^{37-40}$ the potential for misdiagnosis in the 3 patients who underwent needle biopsy exists, in particular with 
respect to differentiation from oncocytic carcinomas. Judicious follow-up of these 3 patients is suggested, with a view for further imaging and repeat biopsy if malignancy becomes a concern.

Although an equal number of 2 parotid oncocytomas with nonenhancing curvilinear clefts were detected by each of the 4- and 64-section Toshiba systems which used comparable time delays in our study, the authors recognize that the difference in the speed of image acquisition between the 2 imaging systems may result in the representation of different phases of tumor enhancement and alter the conspicuity of the nonenhancing curvilinear cleft. In a small series of major salivary gland tumors that included a single submandibular oncocytoma, Kei and $\operatorname{Tan}^{41}$ also described how weak tumor enhancement in an early-phase postcontrast CT scan could potentially produce an attenuation value similar to that of the surrounding native parotid gland parenchyma and make the tumors inconspicuous. We suggest that a further study should be undertaken to examine the conspicuity of parotid oncocytomas and the nonenhancing curvilinear cleft described in our series using dynamic contrast-enhanced CT imaging.

\section{Conclusions}

A combined clinical, radiologic, and pathologic assessment of a patient who presents with a parotid mass is essential in establishing an accurate diagnosis. The CT findings of a nonenhancing curvilinear cleft and the deformable appearance of parotid oncocytomas described in this largest imaging series to date are potentially helpful in distinguishing these benign lesions from other parotid tumors in clinical scenarios that preclude surgical resection or when biopsy results are not diagnostic. Further studies are advocated to validate the specificity and positive predictive value of these imaging features.

\section{References}

1. Hamperl H. Benign and malignant oncocytoma. Cancer 1962;15:1019-27

2. Lee YY, Wong KT, King AD, et al. Imaging of salivary gland tumors. Eur J Radiol 2008;66:419-36

3. Barnes L, Eveson JW, Reichart P, et al, eds. World Health Organization Classification of Tumors: Pathology and Genetics of Head and Neck Tumors. Lyon, France: International Agency for Research on Cancer Press; 2005:237,268

4. Capone RB, Ha PK, Westra WH, et al. Oncocytic neoplasm of the parotid gland: a 16-year institutional review. Otolaryngol Head Neck Surg 2002;126:657-62

5. Politi M, Toro C, Cian R, et al Multifocal adenomatous oncocytic hyperplasia of the parotid gland. Oral Oncol Extra 2005;41:183-87

6. Watson D, Fu YS, Canalis RF. Transitional features of benign and malignant oncocytic tumors: report of two cases. Am J Otolaryngol 1996;17:335-39

7. Gray SR, Cornog JL, Seo IS. Oncocytic neoplasms of salivary glands. Cancer 1976;38:1306-17

8. Blanck C. Eneroth CM, Jakobsson PA. Oncocytoma of the parotid gland: neoplasm or nodular hyperplasia? Cancer 1970;25:919-25

9. Thompson LD, Wenig BM, Ellis GL. Oncocytomas of the submandibular gland. Cancer 1996;78:2281-87

10. Wakely Jr PE. Oncocytic and oncocyte-like lesions of the head and neck. Ann Diagn Pathol 2008;12:222-30

11. Dardick I, Birek C, Lingen MW, et al. Differentiation and the cytomorphology of salivary gland tumors with specific reference to oncocytic metaplasia. Oral Surg Oral Med Oral Pathol Oral Radiol Endod 1999;88:691-701
12. Loretti A, Sturia M, Gentileschi S, et al. Diffuse hyperplastic oncocytosis of the parotid gland. Br J Plast Surg 2002;55:151-52

13. Schwartz IS, Feldman M. Diffuse multinodular oncocytoma of the parotid gland. Cancer 1969;23:636-40

14. Lee SC, Roth LM. Malignant oncocytoma of the parotid gland. Cancer 1976;37:1607-14

15. Özcan C, Talas D, Görür K, et al. Incidental deep lobe parotid gland oncocytic neoplasms in an operated larynx cancer patient. Oral Oncol Extra 2006;42:235-40

16. Shellenberger TD, Williams MD, Clayman GL, et al. Parotid gland oncocytosis: CT findings with histopathologic correlation. AJNR Am J Neuroradiol 2008;29:734-36

17. Howlett DC, Kesse KW, Hughes DV, et al. The role of imaging in the evaluation of parotid disease. Clin Radiol 2002;57:692-701

18. Kim KH, Sung MW, Yun JB, et al. The significance of CT scan or MRI in the evaluation of salivary gland tumors. Auris Nasus Larynx 1998;25:397-402

19. Sakai E, Yoda T, Shimamoto H, et al. Pathologic and imaging findings of an oncocytoma in the deep lobe of the left parotid gland. J Oral Maxillofac Surg 2003;32:563-65

20. Araki Y, Sakaguchi R. Synchronous oncocytoma and Warthin's tumor in the ipsilateral parotid gland. Auris Nasus Larynx 2004;31:73-78

21. Kasai T, Motoori K, Hanazawa T, et al. MR imaging of multinodular bilateral oncocytoma of the parotid gland. Eur J Radiol 2007;63:97-100

22. Divi V, Fatt MA, Teknos TN, et al. Use of cross-sectional imaging in predicting surgical location of parotid neoplasms. J Comput Assist Tomogr 2005;29:315-19

23. Brandwein MS, Huvos AG. Oncocytic tumors of major salivary glands: a study of 68 cases with follow-up of 44 patients. Am J Surg Pathol 1991;15:514-28

24. Kandiloros D, Segas J, Papadimitriou K, et al. Malignant oncocytoma of the parotid with oncocytic change of the contralateral gland. Am J Otolaryngol 1995; 16:200-04

25. Sugimoto T, Wakizona S, Uemura T, et al. Malignant oncocytoma of the parotid gland: a case report with an immunohistochemical and ultrastructural study. J Laryngol Otol 1993;107:69-74

26. Hagino $\mathrm{K}$, Tsunoda $\mathrm{A}$, Ishihara $\mathrm{A}$, et al. Oncocytoma in the parotid gland presenting a remarkable increase in fluorodeoxyglucose uptake on positron emission tomography. Otolaryngol Head Neck Surg 2006;134:708-09

27. Shah VN, Branstetter BF. Oncocytoma of the parotid gland: a potential falsepositive finding on 18F-FDG PET. AJR Am J Roentgenol 2007;189:W212-14

28. Choi DS, Na DG, Byun HS, et al. Salivary gland tumors: evaluation with twophase helical CT. Radiology 2000;214:231-36

29. Yerli H, Teksam M, Aydin E, et al. Basal cell adenoma of the parotid gland: dynamic CT and MRI findings. Br J Radiol 2005;78:642-45

30. Lin CC, Tsai MH, Huang CC, et al. Parotid tumors: a 10-year experience. Am J Otolaryngol 2008;94:94-100

31. Lev MH, Khanduja K, Morris PP, et al. Parotid pleomorphic adenomas; delayed CT enhancement. AJNR Am J Neuroradiol 1998;19:941-66

32. Chawla AJ, Tan TY, Tan GJS. Basal cell adenomas of the parotid gland: CT scan features. Eur J Radiol 2006;58:260-65

33. Kakimoto N, Gamoh S, Tamaki J, et al. CT and MR images of pleomorphic adenoma in major and minor salivary glands. Eur J Radiol 2009;69:464-72

34. Takashima S, Sone S, Honjho Y, et al. Warthin's tumor of the parotid gland with extension into the parapharyngeal space. Eur J Radiol 1997;24:227-29

35. Mafee MF, Venkatesan TK, Ameli MN, et al. Tumors of parotid gland and parapharyngeal space: role of computed tomography and magnetic resonance imaging. Otolaryngol Head Neck Surg 1996;7:348-57

36. Maiorano E, Lo Muzio L, Favia G, et al. Warthin's tumor: a study of 78 cases with emphasis on bilaterality, multifocality and association with other malignancies. Oral Oncol 2002;38:35-40

37. Kesse KW, Manjaly G, Violaris N, et al. Ultrasound-guided biopsy in the evaluation of focal lesions and diffuse swelling of the parotid gland. $\mathrm{Br} \mathrm{J}$ Oral Maxillofac Surg 2002;40:384-88

38. Wan YL, Chan SC, Chen YL, et al. Ultrasonography-guided core-needle biopsy of parotid gland masses. AJNR Am J Neuroradiol 2004;25:1608-12

39. Howlett DC, Menezes LJ, Lewis K, et al. Sonographically guided core biopsy of a parotid mass. AJR Am J Roentgenol 2007;188:223-27

40. Breeze J, Andi A, Williams MD, et al. The use of fine needle core biopsy under ultrasound guidance in the diagnosis of a parotid mass. Br J Oral Maxillofac Surg 2009;47:78-79

41. Kei PL, Tan TY. CT “invisible" lesion of the major salivary glands a diagnostic pitfall of contrast-enhanced CT. Clin Radiol 2009;64:744-46. Epub 2009 May 8 